\title{
5. Neue Möglichkeiten für die Servicerobotik durch KI
}

\begin{abstract}
Steffen Wischmann, Marieke Rohde
Serviceroboter gewinnen an Boden, in professionellen Anwendungen ebenso wie im privaten Bereich. Diesen Vormarsch verdanken sie in erster Linie Fortschritten in der KI, insbesondere den Verfahren des maschinellen Lernens, der Computer Vision und Optimierung, die eine autonomere und adaptivere Handlungssteuerung dieser Roboter auch in einem veränderlichen Umfeld erlauben. So wird ihr Einsatz auch in Bereichen möglich, die klassischen Industrierobotern verschlossen bleiben.
\end{abstract}

Der Begriff Servicerobotik erklärt sich in Abgrenzung zur klassischen Industrierobotik nicht selbst. Die Unterscheidung zwischen beiden ist vielmehr historisch gewachsen. Die meisten Roboter, die heutzutage auf dem Markt verfügbar sind, zählen zu den klassischen Industrierobotern (International Federation of Robotics 2017a), wie sie etwa in der Automobilindustrie flächendeckend das Schweißen oder Lackieren erledigen. Industrieroboter erfüllen normalerweise eine eng begrenzte Aufgabe im Produktionsprozess: Ihr Handlungsrepertoire umfasst meistens nur einen einzigen Prozess, der unter eng definierten Umgebungsbedingungen auszuführen ist. Aus diesem Grund brauchen sie auch nur wenige Sensoren, um sich in ihrem Einsatzbereich zurechtzufinden. Programmierung und Integration dieser Industrieroboter sind allerdings aufwendig und nehmen bislang üblicherweise zwischen 60 und 80 Prozent der gesamten Investitionskosten in Anspruch, da auch kleinste Änderungen der Anwendung oder der Umgebungsbedingungen eine Anpassung der Programmierung erforderlich machen.

Der ISO-Standard 8373 (ISO 2012) listet unter Servicerobotern all jene Robotersysteme, die nicht im vollautomatisierten Umfeld eingesetzt werden. Durch diese Negativdefinition gelten sowohl Roboter im privat-individuellen als auch solche im professionell-beruflichen Kontext - nur eben außerhalb vollautomatischer FertigungsstraBen - als Serviceroboter ${ }^{42}$.

42

Die ISO 8373 unterscheidet in Studien wie der World Robotics (International Federation of Robotics 2017b) dann in einer weiteren Unterteilung zwischen "Personal Service Robots" und "Professional Service Robots": "Personal Service Robots" werden zu nichtkommerziellen Zwecken genutzt und ihre Bedienung erfordert keine besonderen Kenntnisse. Oftmals ist sogar eine Bedienung durch Laien möglich. "Professional Service Robots" werden zu kommerziellen Zwecken genutzt und ihre Bedienung erfordert in der Regel entsprechend geschultes Personal. 
Anders als fest in einem Produktionsprozess installierte Industrieroboter müssen Serviceroboter in einer sich ständig ändernden Umgebung agieren können. Sie müssen deshalb dazu in der Lage sein, zu lernen, sich anzupassen und Fehler autonom zu korrigieren. Serviceroboter haben daher in der Regel auch deutlich mehr Sensoren als Industrieroboter. Da die Genauigkeit und Zuverlässigkeit von Sensordaten häufig stark variieren, besteht für die Kontrollsysteme eines Serviceroboters die schwierige Herausforderung, ein zutreffendes Bild der Umgebung zu generieren, damit Aktionsplanung und -steuerung flexibel auf alle Änderungen reagieren können.

In vielen Bereichen - insbesondere in der Logistik, Medizin, Pflege, Landwirtschaft, Inspektion und Wartung - ist ein Trend zum professionellen Einsatz von Servicerobotern erkennbar. Die entsprechende Hardware steht bereits zur Verfügung. Jedoch sind existierende Lösungen oft immer noch in ihrer Funktionalität stark eingeschränkt. Eine Anpassung des Verhaltens an neue Bedingungen ist heute noch, ähnlich wie bei der klassischen Industrierobotik, mit hohen Systemintegrationskosten verbunden.

In der Servicerobotik gilt die Programmierung von adaptiven Kontrollsystemen daher zurzeit als größte Herausforderung. Dabei setzen die Entwickler zur Flexibilisierung der Kontrollsoftware auf KI. Es lassen sich sieben KI-Technologiebereiche identifizieren, in denen in den letzten Jahren entscheidende Entwicklungen stattfanden: Computer Vision, ML, Aktionsplanung und Optimierung, Cognitive Modeling, Semantische Technologien, Natural Language Processing (NLP) und Neuromorphic Computing (Seifert et al. 2018, s. auch Einleitung zu Kapitel A Technologie „Entwicklungswege zur $\mathrm{KI}^{\prime \prime}$ ). Insbesondere datengetriebene Verfahren wie maschinelles Lernen (ML), d. h. Verfahren, die aus Beispielen lernen können, können Lösungen zu Problemen finden, die einen hohen Grad an Flexibilität in Wahrnehmung und Handlung erfordern. Die Verfügbarkeit großer Datenmengen (Big Data) und die stark gewachsene Rechenleistung haben diese Flexibilisierung gelernter Lösungen ermöglicht. Wie weitreichend diese KI-Technologien bereits die Robotik prägen, zeigen etwa die Fortschritte im autonomen Fahren.

Moderne KI-Systeme bewegen sich freilich noch immer im Bereich der schwachen oder eingeschränkten KI (weak or narrow Al), die zwar ihre Funktionalität in einem abgesteckten Bereich verallgemeinern, nicht jedoch auf neue, unvorhergesehene Probleme übertragen kann. Auch wenn moderne, datengetriebene KI-Verfahren sehr viel flexibler etwa mit veränderlichen Umgebungsbedingungen und Rauschen in Sensordaten umgehen können, sind sie noch weit von den generellen Fähigkeiten eines Menschen, Probleme zu lösen, entfernt. 


\section{Marktpotenzial und aktuelle Entwicklungen in der Servicerobotik}

Die Servicerobotik ist ein Wachstumsmarkt. Die International Federation of Robotics (IFR) schätzt, dass zwischen 2018 und 2020 weltweit knapp 400.000 Serviceroboter im professionellen Bereich und knapp 43 Millionen Serviceroboter im Endkonsumentenmarkt zum Einsatz kommen werden und dass in dieser Zeit in den beiden Segmenten knapp 23 Milliarden Euro bzw. knapp 16 Milliarden Euro umgesetzt werden (International Federation of Robotics 2017b). Laut IFR bedeutet dies für die kommenden Jahre Wachstumsraten im zweistelligen Prozentbereich.

Von insgesamt ca. 7 Milliarden Euro erzielten Umsätzen im Jahr 2017 hat der professionelle Bereich, bezogen auf den Gesamtmarkt, einen Anteil von 62 Prozent (ca. 4,4 Milliarden Euro Umsatz, ca. 80.000 verkaufte Einheiten) und der Endkonsumentenbereich einen Anteil von 38 Prozent (ca. 2,6 Milliarden Euro, ca. 8,6 Millionen Einheiten).

Betrachtet man die Marktabschätzungen zur Servicerobotik im gesamten professionellen zivilen Bereich (inkl. produzierender Industrie), kristallisieren sich drei Anwendungsbereiche heraus, die ein deutlich höheres Marktpotenzial versprechen als der Rest (siehe Abbildung 5.1). Die höchsten Umsätze wurden im Jahr 2017 im Gesundheitswesen (Diagnostik, Chirurgie, Therapie und Rehabilitation) mit knapp 1,6 Milliarden Euro erzielt. Dominiert wird dieser Bereich von der robotergestützten Chirurgie - ca. 70 Prozent aller verkauften Einheiten fallen in diese Kategorie. Die Feldrobotik und Logistik zeichnen sich durch ähnlich hohe Marktanteile aus mit jeweils ca. eine Milliarde Euro im Jahr 2017, wobei der Logistik ein leicht stärkeres Wachstum in den nächsten Jahren vorhergesagt wird. Umsatzmotor in der Feldrobotik waren 2017 ganz klar Melkroboter (ca. 83 Prozent aller verkauften Einheiten in diesem Segment) und in der Logistik fahrerlose Transportfahrzeuge (FTF) außerhalb des Fertigungsbereichs (ebenfalls ca. 83 Prozent aller verkauften Einheiten in diesem Segment).

Zusammen ergeben diese drei spezifischen Roboterarten Chirurgieroboter, Melkroboter und FTF etwa die Hälfte aller abgesetzten Servicerobotik-Einheiten im professionellen Bereich. Alle drei Anwendungsfälle verbindet, dass sie von der Aufgabenstruktur noch der klassischen Industrierobotik ähneln, also die Anforderungen an flexible Wahrnehmung und Handlungssteuerung noch überschaubar sind. Chirurgieroboter werden fast vollständig von Ärzten ferngesteuert, Melkroboter unterscheiden sich von klassischen Melkmaschinen nur durch das automatische Andocken am Euter der Kuh, und FTF fahren feste Routen in Industriegebäuden ab, in denen sich die Sichtverhältnisse kaum ändern.

Das projizierte Wachstum hingegen wird auf neue Anwendungsfälle zurückzuführen sein, die einen höheren Grad an Autonomie erfordern und in denen die KI ihre flexibilisierendes Potenzial voll entfalten kann. 


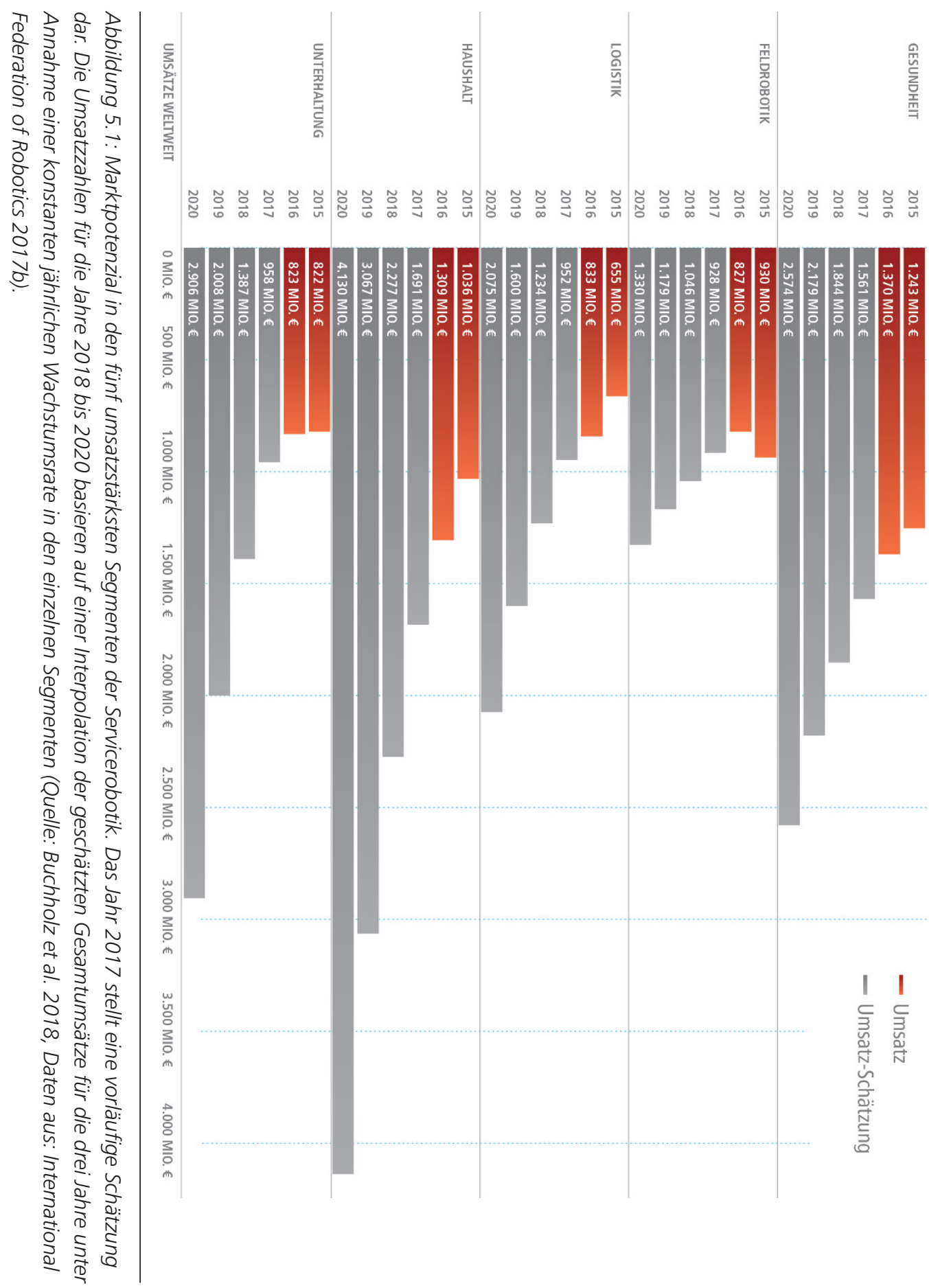




\section{Start-up-Investitionen als Maß für Marktreife}

Das Zusammenspiel von KI-Methoden mit robotischen Systemen ist bereits seit den 1950er Jahren Gegenstand der Forschung. Die Anzahl an wissenschaftlichen Publikationen mit $\mathrm{KI}$ und Robotik im Fokus hat seitdem exponentiell zugenommen (siehe Abbildung 5.2). Deutschland ist nach den USA das publikationsstärkste Land, wenngleich mit großem Abstand.

Die kommerzielle Umsetzung der Forschungsergebnisse in Kl-gesteuerte RobotikProdukte befindet sich demgegenüber noch immer in den Kinderschuhen. Das Verhalten von Investoren im Start-up-Bereich kann hier als Indikator für die Marktreife herangezogen werden, da Start-ups oftmals als erste mit neuen Technologien in den Markt gehen. Erste zaghafte Investitionen in Robotik-Start-ups mit KI-Bezug sind erst seit 2011 zu verzeichnen. Seitdem lässt sich hier allerdings ein exponentieller Anstieg erkennen. Im Jahr 2017 wurden bereits knapp zwei Milliarden US-Dollar in KI-Robo-

\section{Anzahl der Publikationen weltweit}
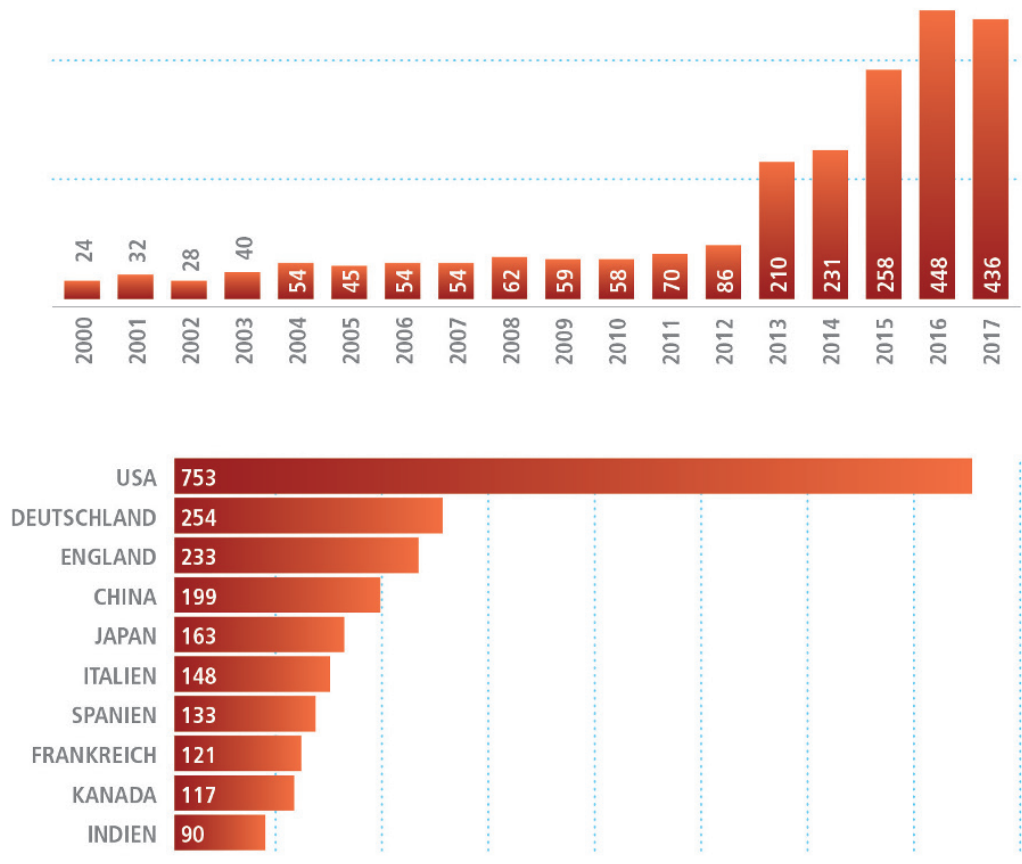

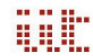

Abbildung 5.2a: Anzahl an wissenschaftlichen Publikationen zu den Themen Kl und Robotik (eigene Darstellung, Daten aus Web of Science, Suchabfragebedingung: TS=(, artificial intelligence" $O R$ "machine learning") AND TS=(robot*)). 


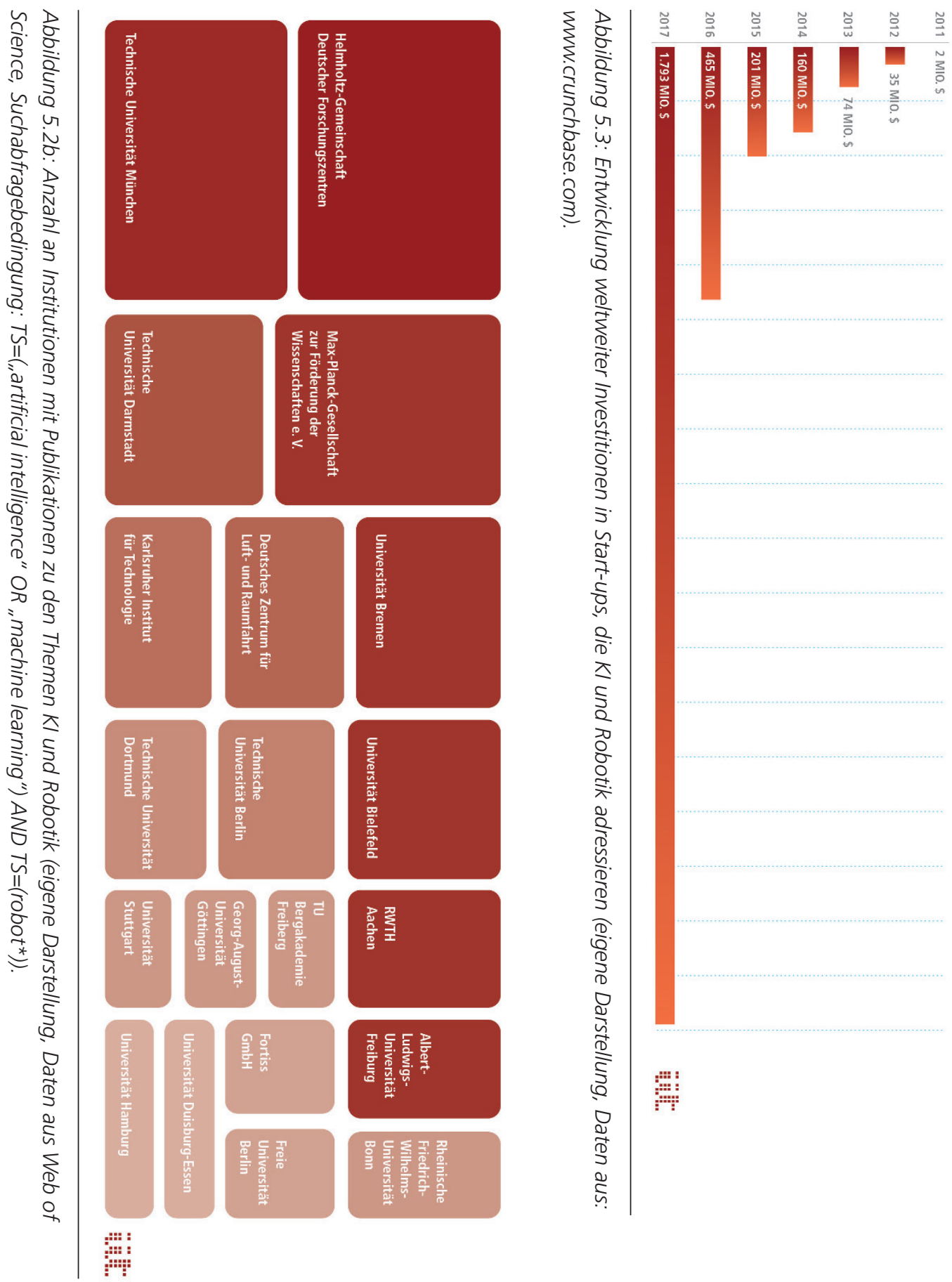




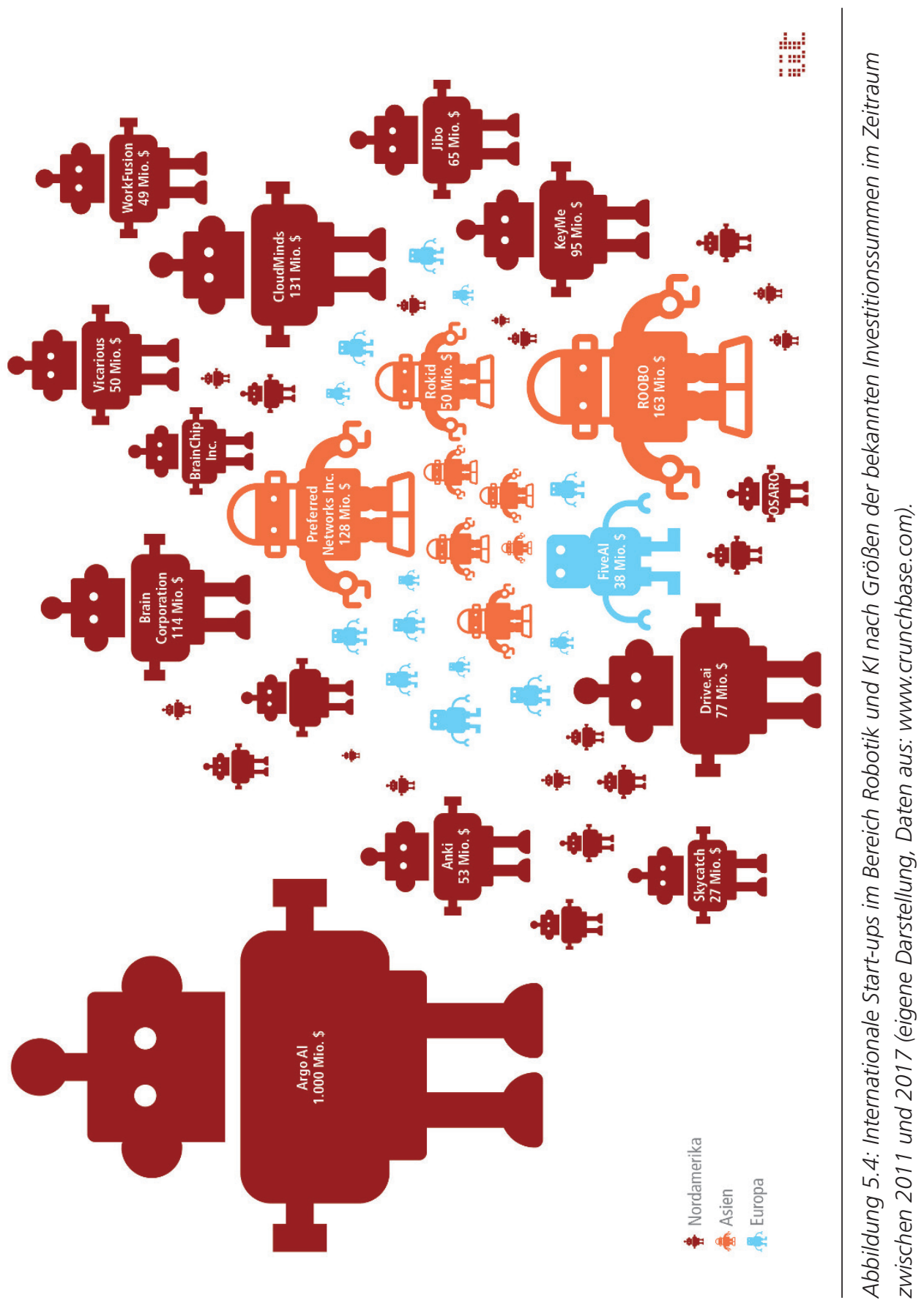


Verteilung von Investitionen in Start-ups in Europa
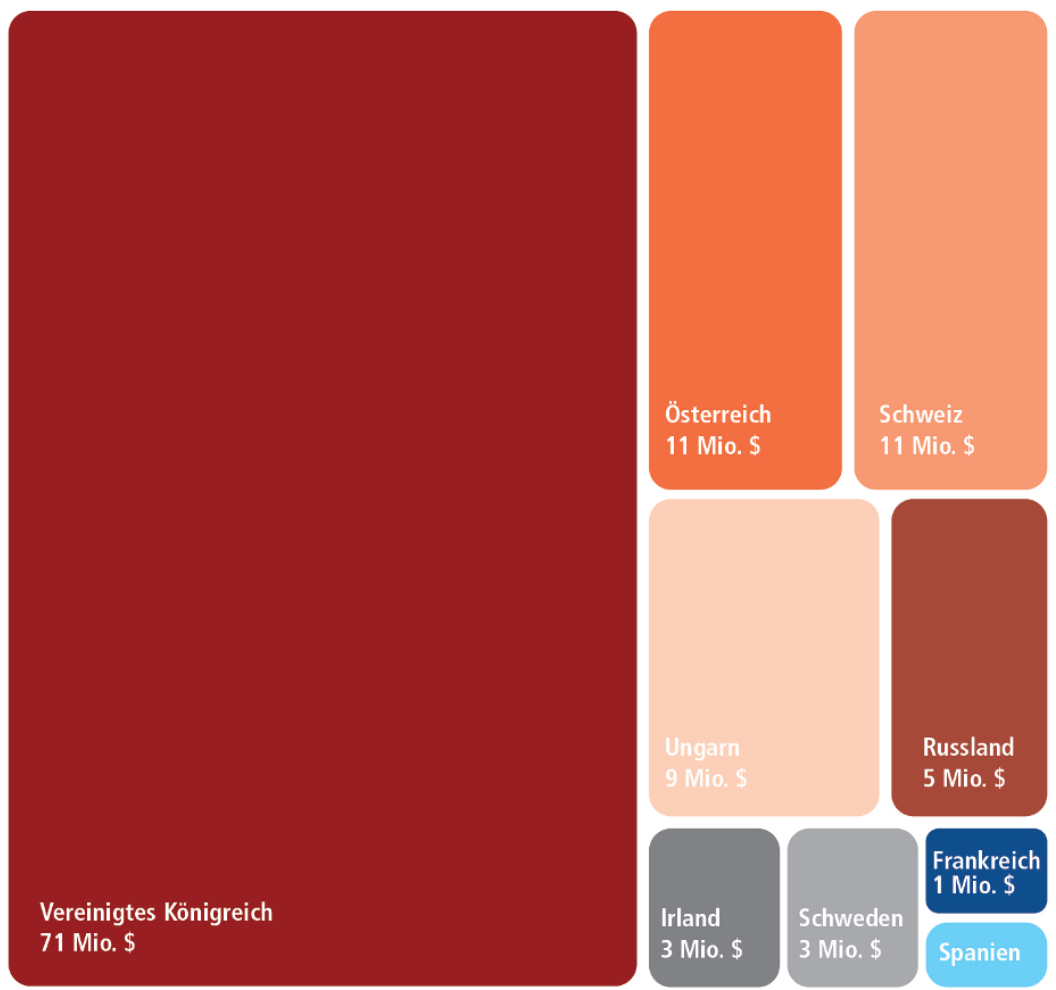

Abbildung 5.5a: Europäische Start-ups im Bereich Robotik und KI: Verteilung der bekannten Investitionssummen nach Ländern (eigene Darstellung, Daten aus: www.crunchbase.com, betrachteter Zeitraum: 2011-2017).

tik-Start-ups investiert (siehe Abbildung 5.3). Im internationalen Vergleich wird die Start-up-Szene vom nordamerikanischen und dem asiatischen Raum dominiert (siehe Abbildung 5.4). ${ }^{43}$

Der Hauptfokus der erfolgreichsten Start-ups liegt auf der Anwendung von KI für autonomes Fahren wie z. B. Argo Al oder Brain Corporation und EndkonsumentenRoboter für Haushalt und Entertainment wie etwa ROOBO, Rokid und Anki. Aller-

43 Berücksichtig wurden alle Einträge der auf www.crunchbase.com verzeichneten Investitionsrunden zwischen 2011 und 2017 von Start-ups, die den Kategorien "Artificial Intelligence" und "Robotics" zugeordnet sind. Datum der Abfrage: 21.02.2018) 


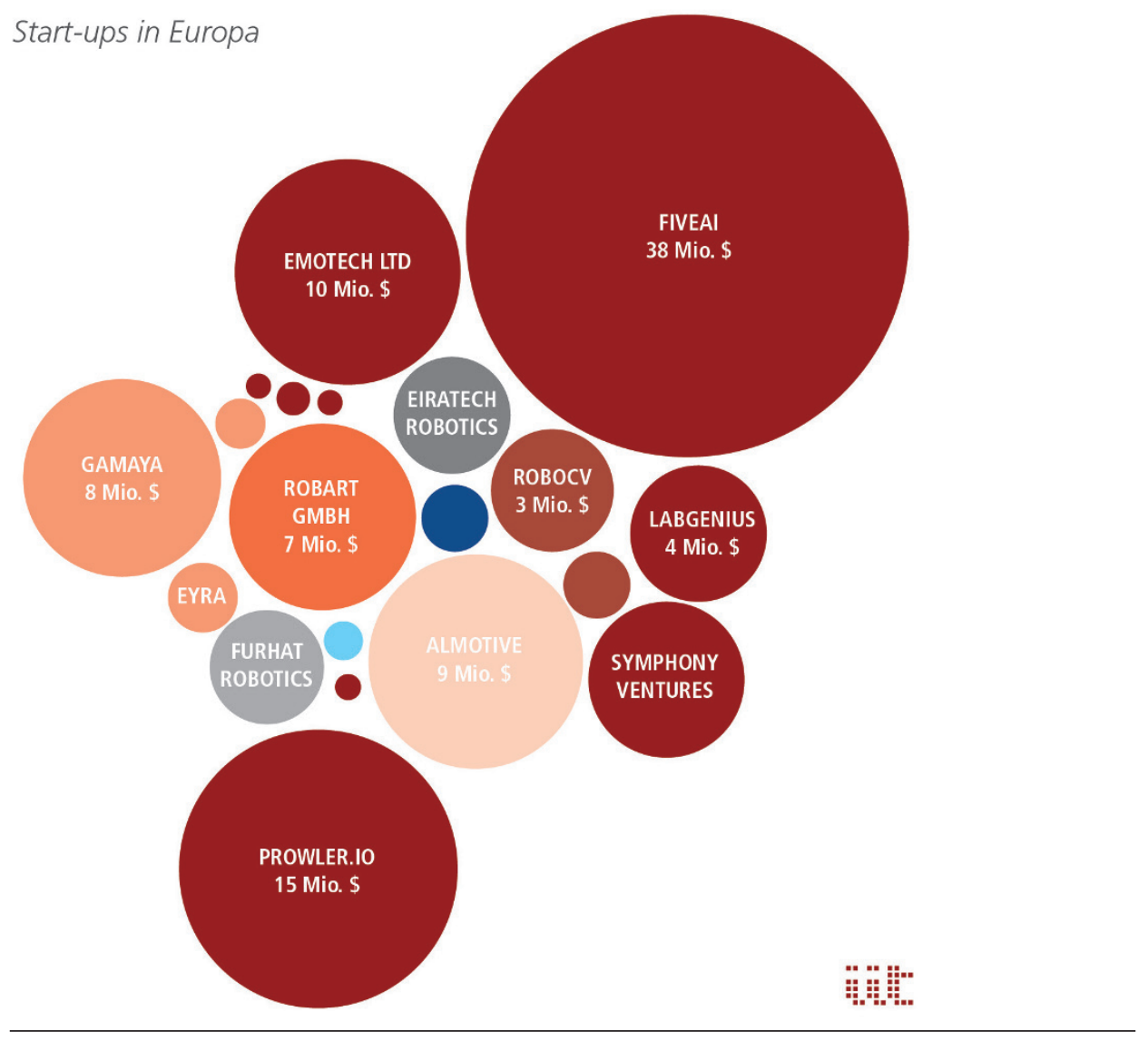

Abbildung 5.5b: Europäische Start-ups im Bereich Robotik und Kl: Start-ups nach Größen der bekannten Investitionssummen (eigene Darstellung, Daten aus: www.crunchbase.com, betrachteter Zeitraum: 2011-2017).

dings finden sich auch erste Start-ups mit hohen Investitionssummen im Umfeld der Produktion (z. B. Preferred Networks).

Im europäischen Vergleich fallen die Investitionssummen deutlich geringer aus. Britische Start-ups führen das Feld in Europa an, deutsche hingegen verzeichneten bis Ende 2017 noch keine nennenswerten Investitionssummen (siehe Abbildung 5.5). Dass das deutsche Logistikroboter-Start-up Magazino Anfang 2018 eine Finanzierungsrunde in Höhe von 20,1 Millionen Euro abschloss ${ }^{44}$ ist in hier noch nicht erfasst.

44 https://www.handelsblatt.com/unternehmen/industrie/lagerroboter-zalando-steigt-beivorzeige-start-up-magazino-ein/21007764.html, zuletzt geprüft am 22.06.2018 
Auch europäische Start-ups konzentrieren sich auf das autonome Fahren wie z. B. Five Al oder Almotive und Haushalts- und Entertainmentroboter für den Endverbraucher (z. B. Emotech, Furhat Robtics). Andere europäische Start-ups entwickeln Kerntechnologien. So widmet sich beispielsweise Prowler.IO dem „decision making”, einer Grundvoraussetzung für die Aktionsplanung, während andere Unternehmensneugründungen Computer Vision-Technologien für die Robotik erarbeiten. Jedoch gibt es bereits auch vereinzelte Start-ups, die konkrete Produkte in anderen Anwendungsbereichen konzipieren. Gamaya beispielsweise findet Robotiklösungen für den landwirtschaftlichen Sektor, Robart konzentriert sich auf Navigation von Reinigungsrobotern, und EiraTech, Magazino und RoboCV entwickeln Robotikprodukte für die Intralogistik.

Die Diskrepanz bei den Start-up-Investitionen zwischen Kontinentaleuropa, dem englischsprachigen Raum (Großbritannien und insbesondere den USA) und Asien belegt zum einen, dass Deutschland und anderen Ländern Kontinentaleuropas wie in den meisten Bereichen angewandter digitaler Innovation eine Außenseiterrolle zukommt. Andererseits belegen der Anstieg von Start-up-Investitionen weltweit und die zunehmende Diversifizierung der Anwendungsfälle, dass KI-Innovationen der Robotik tatsächlich neue Märkte und Anwendungsszenarien eröffnen, und dass dieses Marktpotenzial auch in Europa genutzt wird.

Der Innovationshorizont für Kl-getriebene Robotik lässt sich an Beispielen aus der Landwirtschaftsrobotik (Feldrobotik) sowie der Medizin- und Pflegerobotik erkennen. Ein Vergleich dieser beiden Bereiche ist überaus interessant: Einerseits haben die aktuellen Servicerobotik-Hardwareplattformen bereits in beiden Bereichen einen sehr hohen technologischen Reifegrad erreicht. Allerdings leidet deren breite Anwendung noch an einem Mangel an zuverlässigen Algorithmen für die intelligente Sensordatenverarbeitung und adaptive Steuerung. Hier kann die moderne KI ihre flexibilisierende Funktion wahrnehmen. Anderseits stehen beide Bereiche vor teilweise sehr unterschiedlichen Herausforderungen. So ist die Mensch-Maschine-Interaktion ein zentraler Faktor für die Medizin- und Pflegerobotik, nicht jedoch für die autonome Feldrobotik.

\section{Intelligente Roboter in der Landwirtschaft}

Während autonome Fahrzeuge erst zögerlich im Straßenverkehr auftauchen, sind auf den Feldern dieser Welt schon seit Jahren halbautonom gesteuerte Landwirtschaftsmaschinen unterwegs. Dank GPS, optischen Sensoren und ausgefeilter Regelungstechnik können sie einer vorgegebenen Spur automatisch folgen - auf wenige Zentimeter genau. Allerdings sind solche Hightech-Landmaschinen bislang nur für Großbetriebe rentabel. Sie sind sehr groß und sehr teuer und können nur einfache, isolierte Arbeitsschritte wie z. B. Mähdreschen und Säen ausführen, die kein weiteres autonomes Verhalten erfordern (International Federation of Robotics 2017b). 
Dank neuer KI-Technologien finden jetzt jedoch auch kleinere und vielfältiger einsetzbare Serviceroboter den Weg in die Landwirtschaft (Wölbert 2017). Diese leichten und kostengünstigeren Feldroboter profitieren insbesondere von den KI-Technologien Computer Vision, ML sowie Aktionsplanung und Optimierung (siehe Abbildung 5.6), die eine autonome Navigation auf dem Acker überhaupt erst möglich machen. Von dieser Entwicklung profitieren auch kleinere Landwirtschaftsbetriebe, und der Trend zu kleinen Feldrobotern könnte diese Maschinen sogar bald auch attraktiv für die Bewirtschaftung von privaten Gärten machen (Joe Jones 2017).

Das Aufgabenspektrum, das die neuen KI-getriebenen Feldroboter bereits heute erledigen und künftig noch besser werden meistern können, wächst von Tag zu Tag. So werden Drohnen genutzt, um optische Aufnahmen vom Feld zu machen, auf denen mit Hilfe von Computer Vision-Algorithmen relevante Objekte wie Früchte, Schädlinge und Unkraut) und Parameter (Pflanzendichte) detektiert werden können (z. B. Lottes et al. 2017). Fortschritte in der Aktionsplanung ermöglichen präzise

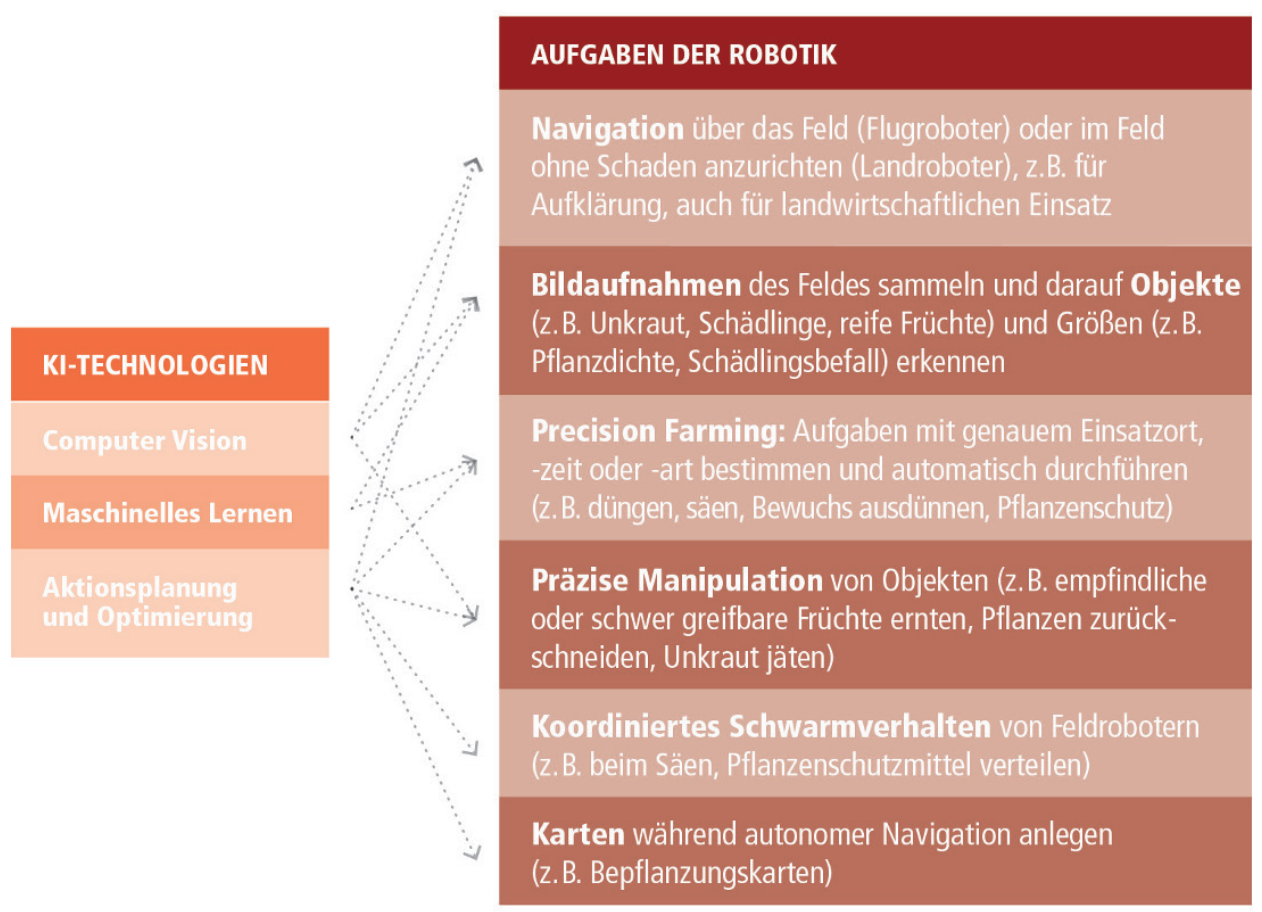

B:

Abbildung 5.6: Neue KI-Technologien eröffnen der Landwirtschaftsrobotik neue Aufgabenfelder. 
Manipulationen wie das Ernten von empfindlichen Früchten (van Henten et al. 2002) oder das Zuschneiden von Nutzpflanzen (Ackermann 2012). Und schließlich können Feldroboter ihre Aktivitäten mitschreiben und z. B. Bepflanzungskarten automatisch generieren.

Der Landwirtschaft stehen inzwischen sehr umfangreiche Sensordaten über die aktuellen Zustände auf den Feldern zur Verfügung, die von KI-Algorithmen ausgewertet optimale Handlungsentscheidungen ermöglichen (Sfiligo 2016). Ein Beispiel für solches "Precision Farming" ist die Installation von Stickstoffsensoren, die regelmäßig Daten über den lokalen Zustand des Bodens übermitteln. Daraus lässt sich ein gezieltes Düngen ableiten, ggf. punktuell nur dort, wo ein Stickstoffmangel festzustellen ist. Das senkt Kosten (weniger Dünger), ist effektiver (Überdüngung wirkt sich negativ auf den Ertrag aus) und schont nebenbei auch die Umwelt (Adamchuk et al. 2004). Moderne datengetriebene KI-Verfahren zur Aktionsplanung und Optimierung können neben Sensordaten vom Feld auch Vorwissen wie Bepflanzungskarten und externe Datenquellen wie z. B. Wetterdaten in die Entscheidungsfindung miteinbeziehen und damit die Landwirtschaft noch effektiver, präziser und wirtschaftlicher machen. Autonomen Feldrobotern kommt hierbei die wichtige Rolle zu, die einzelnen Handlungsschritte automatisch sowie zeitlich und örtlich präzise umzusetzen.

Bei kleinen Feldrobotern werden auch Roboterschwärme erforscht (King 2017) oder sogar schon angewandt (Wölbert 2017). Auch dies macht die autonome Feldrobotik attraktiver für kleine Landwirtschaftsbetriebe, die in Gruppen oder Kooperativen einzelne Landwirtschaftsroboter kaufen können, um sie dann für flächendeckende Einsätze, z. B. für die Saat, zu Schwärmen zusammenzufügen (King 2017). Die Koordination von Gruppen autonomer Roboter ist eine weitere Herausforderung für die KI in Aktionsplanung und Optimierung.

Neben dem beschriebenen Trend zu kleineren autonomen Feldrobotern wird auch eine zunehmende Vielseitigkeit der Funktionalität von Feldrobotern kleinen Landwirtschaftsbetrieben zugutekommen. Fortschritte in KI-Technologien wie ML machen es möglich, ein und dieselbe Roboterplattform auf mehrere landwirtschaftliche Aufgaben zu trainieren, etwa das Ernten verschiedener Früchte und das Versprühen von Pflanzenschutzmitteln (van Henten et al. 2002; Tobe 2014). Die Anschaffung eines autonomen Feldroboters, der verschiedene Arbeiten bewältigen kann, wird sich schneller rentieren.

Die Beispiele zeigen, dass die durch KI erweiterte Flexibilität und erhöhte Autonomie von Feldrobotern immer noch überschaubar ist - zumal, wenn man sie mit der Fähigkeit von Menschen, Probleme zu lösen, vergleicht. KI-Verfahren und autonome Feldroboter können menschliche Entscheidungen optimieren und eine ständig wachsende Bandbreite von in der Landwirtschaft notwendigen Handlungsschritten autonom ausführen. Den Menschen als Entscheider ersetzen können sie jedoch in keiner Weise. 


\section{Intelligente Roboter in Medizin und Pflege}

Im Unterschied zur Landwirtschaft warten in Medizin und Pflege Herausforderungen ganz anderer Art auf KI und Robotik, denn hier sollen autonome Roboter mit Menschen interagieren können. Selbstverständlich gibt es auch in diesem Bereich Einsatzfälle einfacherer Art, z. B. in der Gebäudereinigung, der Entsorgung von medizinischem Müll oder der Organisation medizinischer Güter (International Federation of Robotics 2017b). Solche Anwendungen unterscheiden sich jedoch wenig von anderen Aktivitäten in der Logistik generell. Hier liegt der Fokus daher auf Servicerobotern, die mit Menschen interagieren.

Roboter können Menschen auf einer rein körperlichen Ebene behandeln, wie bei der Präzisionschirurgie, der intelligenten Prothetik oder auch bei chirurgischen Schulungen. Dabei erstreckt sich die Autonomie der Roboter hauptsächlich auf das Bereinigen und Korrigieren, etwa zur Stabilisierung der menschlichen Bewegungen (International Federation of Robotics 2017b). Relevant für diese Anwendungsfälle sind hauptsächlich die KI-Technologien ML, Computer Vision, Aktionsplanung und Optimierung.

Technologisch anspruchsvoller sind Systementwicklungen, die eine Mensch-RoboterInteraktion beinhalten. Hierbei soll etwa ein Assistenz- oder Rehabilitationsroboter als autonomer Agent mit einem Menschen kooperieren (International Federation of Robotics 2017b). Zu unterscheiden sind dabei die physische Interaktion (Ikemoto et al. 2012), in der Mensch und Roboter einander berühren oder sich zusammen bewegen, und der verbale Austausch, bei dem Roboter und Mensch sich auch sprachlich verständigen. In der Mensch-Roboter-Interaktion ermöglichen neue KI-Methoden eine Flexibilisierung der sensorischen Datenverarbeitung (Wahrnehmung), der Aktionsplanung und der Handlung. Zusätzlich zur Umgebung muss auch der Mensch als Akteur modelliert und interpretiert werden: menschliches Wohlbefinden wie Emotionen oder Gesundheit und menschliche Ziele wie Intentionen gilt es aus den Sensordaten abzuschätzen und durch robotische Handlungen zu unterstützen. Auf sich schnell ändernde Bewegungen und Reaktionen des Menschen muss der Roboter in Echtzeit eingehen. Hierfür bedarf es neben Computer Vision, ML, Aktionsplanung und Optimierung auch neuer KI-Technologien in den Bereichen Kognitive Modellierung, Natural Language Processing (NLP) und Semantische Technologien (siehe Abbildung 5.7).

In Japan, wo die Akzeptanz für robotische Assistenzsysteme sehr viel höher ist als in Deutschland, werden in der Altenpflege schon seit mehr als zehn Jahren robotische Haustiere eingesetzt, die sehr einfache Verhaltensschaltkreise haben (Rabe und Kohlbacher 2015). Auch in anderen Bereichen können soziale robotische Assistenzsysteme hilfreich sein, z. B. bei autistischen Kindern, die in manchen Fällen die Interaktion mit einem Roboter der Interaktion mit Menschen vorziehen (Feil-Seifer und 


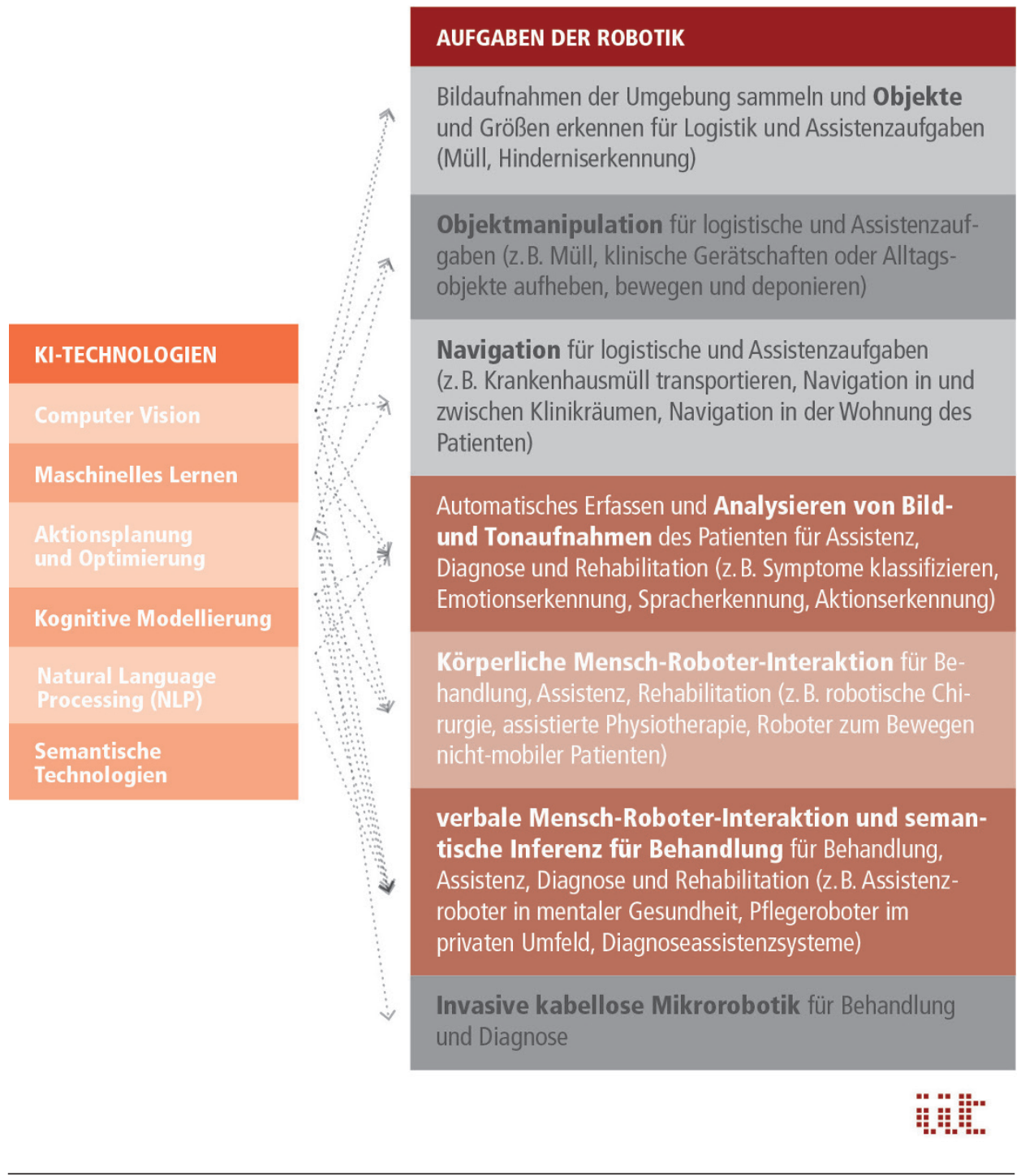

Abbildung 5.7: Neue KI-Technologien (Seifert et al. 2018) eröffnen der Medizin- und Pflegerobotik neue Aufgabenfelder. Die hier diskutierten Anwendungsfälle mit Bezug zur Mensch-Roboter-Interaktion sind farblich hervorgehoben. 
Mataric 2011). Bekannt ist zudem, dass Kriegsveteranen sich häufig lieber von einem virtuellen Avatar als von einem Psychotherapeuten behandeln lassen, um der Stigmatisierung ihrer mentalen Krankheit zu entgehen (Lucas et al. 2017).

Solche robotischen "Companion"-Systeme, die Menschen in erster Linie körperlich oder emotional stützen, könnten in Zukunft vermehrt dazu genutzt werden, den Gesundheitszustand eines Patienten zu überwachen. Denn NLP, ML und Computer Vision sind zunehmend dazu fähig, Emotionen, mentale Zustände und Stress im Allgemeinen zu erkennen (El Kaliouby und Robinson 2004), zudem besonders auch Symptome körperlicher (Bartlett et al. 2014) und mentaler (Bedi et al. 2015) Krankheiten einzuschätzen. Roboter-Companions könnten somit die zur Zeit bestehenden Versorgungslücken in der Überwachung von gefährdeten Patientinnen und Patienten schließen, da sie, im Gegensatz zu menschlichem Pflegepersonal, rund um die Uhr im vollen Umfang einsatzfähig sind und z. B. automatisch Alarm auslösen können, wenn sie einen kritischen Zustand erkennen.

Auf Basis von KI-Technologien zur intelligenten Interpretation solcher diagnoserelevanten Sensordaten könnten robotische Assistenzsysteme künftig auch direkt selbst eingreifen, sei es, dass sie Patienten daran erinnern, bestimmte Aktionen durchzuführen wie etwa Medikamente einzunehmen, oder dass sie mit dem Patienten zusammenarbeiten. So könnten Rehabilitationsroboter - ähnlich einem "Serious Gaming"-Ansatz - einem Patienten motivierendes Feedback zu dessen Verhalten geben. Auch könnten Roboter Aufgaben übernehmen, die für menschliche Pfleger unangenehm sind, gesundheitsgefährdend oder sie gar überfordern, wie das Heben oder Bewegen nichtmobiler Patientinnen und Patienten (International Federation of Robotics 2017b).

Je interaktiver eine geplante Roboteraufgabe ist, desto höher sind die Anforderungen an die KI-Technologien, die menschlichen Aktionen und Intentionen nachzuvollziehen und auf Reaktionen einzugehen. Hier kommen NLP-Technologien eine wichtige Funktion zu, da Roboter, die bei der Schätzung menschlicher Verhaltensabsichten aufgrund von Beobachtung sehr unsicher sind, mittlerweile sogar klärend nachfragen können (Whitney et al. 2017; Ackermann 2017a).

\section{Handlungsräume}

Für die noch relativ junge Servicerobotik kann KI zu einer Schlüsseltechnologie werden, die ihr einen breiten Einsatz in unterschiedlichsten Feldern ermöglicht. Gerade in Deutschland könnten Serviceroboter nicht nur in der Industrie dazu beitragen, die Produktivität zu steigern und nachhaltiger zu wirtschaften, sondern auch den demografischen Wandel ausgleichen und außerhalb der Fabrikhallen in zentralen Bereichen unserer Gesellschaft entlastend wirken. 
In der Pflege besteht die Hoffnung, dass repetitive und physisch anstrengende Tätigkeiten in absehbarer Zeit auf Serviceroboter übertragen werden können. Als Companions könnten Serviceroboter eine kontinuierliche Gesundheitsüberwachung von pflegebedürftigen Personen gewährleisten. Das ohnehin knappe Pflegepersonal könnte sich dann verstärkt den Kernaufgaben wie der Fürsorge widmen; Tätigkeiten also, bei denen für die nächsten Jahrzehnte nicht erkennbar ist, dass KI-Methoden hier auch nur ansatzweise den Menschen ersetzen könnten.

In der Landwirtschaft erhöht ein zunehmender Preisdruck den Bedarf an effizienten Automatisierungsmethoden. Verschärft wird dies auch dadurch, dass das Interesse in der Gesellschaft abnimmt, einen Berufsweg in diesem Wirtschaftszweig zu suchen, sowie durch die Schwierigkeit für Landwirte, überhaupt Personal für Helfertätigkeiten zu finden. Ähnliche Tendenzen zeigen sich auch im Straßenbau oder in der Abfallwirtschaft.

Die Basis für eine schnelle Entwicklung von Servicerobotern als ständige Begleiter des Menschen wurde in der KI-Grundlagenforschung der vergangenen Jahrzehnte gelegt. Die Verfügbarkeit der hierfür benötigten hohen Datenmengen sowie von großer Rechenleistung ist mittlerweile ebenfalls gegeben. Jetzt kommt es darauf an, die KI-Methoden an die unterschiedlichen Anwendungen der Servicerobotik anzupassen.

Beim autonomen Fahren geht heute schon ein rasanter Fortschritt mit steigenden Investitionen einher. Die weitere Beschleunigung der Entwicklung in diesem Anwendungsgebiet bedarf aufgrund des hohen Marktpotenzials nur geringer innovationspolitischer Eingriffe, wie auch die Entwicklungen des Investitionsverhaltens im Startup Bereich unterstreichen. Die Märkte in Landwirtschaft, Medizin und Pflege hingegen sind noch deutlich kleiner und die Anwendungsfälle deutlich heterogener. Hier sind konzentrierte innovationspolitische Interventionen sinnvoll, um die KI-gesteuerte Servicerobotik auf den Weg zu bringen.

Wie in vielen anderen Bereichen angewandter neuartiger digitaler Technologien fehlen auch in der Kl-gesteuerten Servicerobotik junge innovative Unternehmen, um das große Potenzial zu nutzen, das in der KI-Wissenschaft in Deutschland vorhanden ist. Der Kontrast zu den USA und asiatischen Ländern ist enorm, aber auch im europäischen Vergleich hinkt Deutschland deutlich etwa hinter Großbritannien her. Um diese Kluft zu schließen, sind fokussierte innovationspolitische Anreize für den Know-howTransfer von der in Deutschland gut aufgestellten KI- und Robotik-Forschung (EFI 2018; Seifert et al. 2018) in die Wirtschaft notwendig. Dies gilt besonders für Märkte wie Gesundheitsrobotik und Landwirtschaftsrobotik, in denen der Bedarf enorm ist. Die technologischen Voraussetzungen für marktreife Produkte der Servicerobotik sind gegeben. 


\section{Ausblick}

Künstliche Intelligenz und Robotik sind aktuell Megathemen der öffentlichen Debatte. Die tatsächlich signifikanten Durchbrüche in KI-Technologien wie ML und Computer Vision werden in den Medien jedoch gelegentlich zum Ausgangspunkt überbordender Fantasien für übertriebene Projektionen des Möglichen in der Zukunft, in der Roboter - ob zum Guten oder zum Schlechten - bald schon einen Großteil der heute von Menschen ausgeübten Tätigkeiten bewältigen können (Brooks 2017). Dies schürt vor allem Ängste vor massiven Arbeitsplatz- und auch Kontrollverlusten zugunsten digitaler Akteure und Entscheider. Bei kaum einem anderen Technologiethema ist die Kluft zwischen solchen Prophezeiungen und den tatsächlichen Möglichkeiten größer.

Das in den Medien gezeichnete Bild ist deshalb übertrieben und falsch, weil die kolportierten Fortschritte bisher vor allem im Bereich der schwachen oder eingeschränkten $\mathrm{KI}$ erzielt wurden. Moderne KI-Algorithmen funktionieren nur in einem eng abgesteckten Anwendungsfeld. Maschinelle Lernverfahren erkennen Strukturen in Übungsdaten und können auch Lücken füllen, aber sie verstehen nicht die Bedeutung des Gelernten und können ihr Wissen deshalb nicht auf neue, unbekannte Situationen übertragen.

Als beispielsweise der französische Präsident Emanuel Macron seine Wähler mit "Mes chers compatriotes" ("Meine lieben Landsleute") ansprach, wurde dies von Microsofts Bing Übersetzer als "My fellow Americans" (meine amerikanischen Mitbürger) ins Englische übertragen (Bryson 2017). Der KI-Algorithmus hatte gelernt, dass "Landsleute" im englischsprachigen Raum statistisch betrachtet meist "Amerikaner" bedeutet, und verwendet die Worte deshalb als Synonyme. Auch gab es im Jahr 2016 in Florida einen tödlichen Autounfall mit einem autonom gesteuerten Pkw, der mit hoher Geschwindigkeit in einen quer die Straße kreuzenden Sattelschlepper hineinsteuerte, weil das Computer Vision-System diesen nicht erkannt hatte (Greenemeier 2016). Der Hersteller Tesla vermutet, dass das Computer VisionSystem den Sattelschlepper aufgrund seiner weißen Farbe für einen Wolkenhimmel gehalten hat (Greenemeier 2016). Solch ein Fehler hätte einem menschlichen Fahrer nicht passieren können, der weiß, was Hindernisse sind, und deshalb auf jedes Objekt, das sich beim Näherkommen visuell ausdehnt, mit Bremsen reagiert. Diese Beispiele zeigen das fehlende Verständnis des Gelernten bei KI-Systemen auf, was deren Möglichkeiten zur intelligenten Entscheidungsfindung stark einschränkt.

Um die Fähigkeiten einer schwachen $\mathrm{KI}$ auf neue, unvorhergesehen Situationen zu übertragen, müssen also immer noch menschliche Ingenieure Hand anlegen und die Architektur nachbessern oder neue Trainingsdaten bereitstellen. Die Entwicklung von Robotern, die wie Menschen eigenständig, kreativ und flexibel auf neue, unvorhergesehene Situationen reagieren, $\mathrm{d}$. h. die von einer starken oder generellen $\mathrm{KI}$ (strong 
or general Al) gesteuert werden, ist deshalb trotz massiver Fortschritte in maschinellem Lernen noch nicht absehbar (vgl. Brooks 2017).

Deshalb sind auch aufkommende Sorgen in der Bevölkerung, dass Arbeitsplätze an autonome, Kl-gesteuerte Roboter verloren gehen könnten, bis auf wenige Ausnahmen unbegründet. Es gibt keine Evidenz dafür, dass KI, Industrie 4.0, digitale Technologien und Robotik nachhaltig Arbeitsplätze kosten (Graetz und Michaels 2015). Diese Angst hat sich allerdings inzwischen schon festgesetzt und folgt einem historisch bekannten Muster (Mokyr et al. 2015). Kl, Robotik und andere Automatisierungstechnologien vernichten in der Regel keine Arbeitsplätze, sie ersetzen allerdings vorhandene Tätigkeiten (Autor et al. 2003; Spitz-Oener 2006). Dadurch verschieben sich die Arbeitsinhalte von Mitarbeitern oft hin zu anspruchsvolleren Arbeiten (Wischmann und Hartmann 2018).

Es wäre zu begrüßen, wenn sich der öffentliche Diskurs weniger an unrealistischen Bedrohungsszenarien orientiert, sondern die viel weitreichenderen gesellschaftlichen Möglichkeiten der Kl-gesteuerten Servicerobotik ins Auge fassen würde. Wie die angeführten Beispiele zeigen, sind die in fünf bis zehn Jahren zu erwartenden Serviceroboter nicht nur wirtschaftlich gesehen kurz- und mittelfristig vorteilhaft (Erhöhung der Produktivität), sie werden auch zumeist Arbeiten übernehmen, die Menschen entweder nicht übernehmen können oder wollen - unangenehme, schwere oder repetitive Tätigkeiten, Beschäftigungen, die eine durchgängige Aufmerksamkeit und Arbeitsbereitschaft erfordern, oder Arbeiten, deren Präzision jenseits des menschlich Möglichen liegt. Zudem können KI-gesteuerte Serviceroboter einen groBen Beitrag zur Nachhaltigkeit leisten (beispielsweise ökologische Landwirtschaft), von der auch künftige Generationen profitieren werden.

Auch hinsichtlich der langfristigen Perspektiven des Fortschritts in KI und Robotik sind die aktuellen Debatten wahrscheinlich nicht zielführend. Während ein Durchbruch in starker $\mathrm{KI}$ - Voraussetzung für die beschriebenen Angstszenarien - noch in keiner Weise abzusehen ist, werden in Forschung und Entwicklung auf Basis der schwachen $\mathrm{KI}$ andere futuristisch anmutende Anwendungsbeispiele für Roboter untersucht, die völlig neue Mehrwerte für unsere Gesellschaft generieren könnten. Im Bereich Landwirtschaft könnten Roboter beispielsweise Seetangfarmen in den Ozeanen bewirtschaften, deren Ernteertrag etwa zur Erzeugung erneuerbarer Energien genutzt werden könnte (Ackermann 2017b). In der Medizin könnte die noch junge Disziplin der Mikrorobotik es ermöglichen, Krankheiten im Inneren des menschlichen Körpers mit Hilfe sehr kleiner autonomer Roboter gezielt zu behandeln und Lebensprozesse zu erforschen (Sitti et al. 2015). Zu hoffen wäre unter anderem auf völlig neue Perspektiven in der minimalinvasiven Krebstherapie.

$\mathrm{KI}$-Technologien wie Machine Learning und Computer Vision haben das Potenzial, der Servicerobotik neue Anwendungsmöglichkeiten und neue Märkte zu eröffnen. 
Die menschliche Vorstellungskraft bleibt allerdings gern im Rahmen dessen, was aus eigener Erfahrung bekannt ist, und mangelnde Kenntnis des Neuen lässt Fantasien aus der Science-Fiction von Robotern entstehen, die wie Menschen agieren können. Derartige angsterzeugende Szenarien sind unrealistisch und verschleiern das tatsächliche vorhandene enorme Potenzial der KI-gesteuerten Robotik, die Gesellschaft, den Alltag, die Arbeit, die Wirtschaft und die Umwelt positiv zu beeinflussen. Hier gilt es gegenzusteuern. 


\section{Literatur}

Ackermann, Evan (2012): Wall-Ye Robot Is In Your Vineyard, Prunin' Your Vines. A plucky French robot is ready to help out in your vineyard. Online verfügbar unter https:// spectrum.ieee.org/automaton/robotics/industrial-robots/wallye-robot-, zuletzt geprüft am 26.02.2018.

Ackermann, Evan (2017a): Robot Knows the Right Question to Ask When It's Confused. Understanding when they don't understand will help make robots more useful. Online verfügbar unter https://spectrum.ieee.org/automaton/robotics/artificial-intelligence/ robot-knows-the-right-question-to-ask-when-its-confused, zuletzt geprüft am 01.03.2018.

Ackermann, Evan (2017b): Robotic Kelp Farms Promise an Ocean Full of Carbon-Neutral, Low-Cost Energy. Open-ocean kelp farms with drone shepherds could provide renewable energy that doesn't take up land area. Online verfügbar unter https://spectrum.ieee.org/ energywise/energy/renewables/robotic-kelp-farms-promise-an-ocean-full-of-carbon-neutral-low-cost-energy, zuletzt geprüft am 06.03.2018.

Adamchuk, V. I., Hummel, J. W., Morgan, M. T.,; Upadhyaya, S. K. (2004): On-the-go soil sensors for precision agriculture. In: Computers and electronics in agriculture (44(1)), 71-91.

Autor, D. H.; Levy, F.; Murnane, R. J. (2003): The Skill Content of Recent Technological Change. An Empirical Exploration. In: The Quarterly Journal of Economics 118 (4), S. 1279-1333. DOI: 10.1162/003355303322552801.

Bartlett, Marian Stewart; Littlewort, Gwen C.; Frank, Mark G.; Lee, Kang (2014): Automatic decoding of facial movements reveals deceptive pain expressions. In: Current biology : CB 24 (7), S. 738-743. DOI: 10.1016/j.cub.2014.02.009.

Bedi, Gillinder; Carrillo, Facundo; Cecchi, Guillermo A.; Slezak, Diego Fernández; Sigman, Mariano; Mota, Natália B. et al. (2015): Automated analysis of free speech predicts psychosis onset in high-risk youths. In: NPJ schizophrenia 1, S. 15030. DOI: 10.1038/ npjschz.2015.30.

Brooks, Rodney (2017): The Seven Deadly Sins of Al Predictions. Mistaken extrapolations, limited imagination, and other common mistakes that distract us from thinking more productively about the future. (MIT Technology Reviews). Online verfügbar unter https:// www.technologyreview.com/s/609048/the-seven-deadly-sins-of-ai-predictions/, zuletzt aktualisiert am 06.10.2017, zuletzt geprüft am 22.02.2018.

Bryson, Joanna (2017): Why you can't "just fix" machine bias derived from ordinary language (Adventures in NI (persönlicher Blog)). Online verfügbar unter https://joanna-bryson.blogspot.de/2017/05/why-you-cant-just-fix-machine-bias.html, zuletzt aktualisiert am 08.05.2017, zuletzt geprüft am 22.02.2018.

Buchholz, Birgit; Gabriel, Peter; Kraus, Tom; Künzel, Matthias; Richter, Stephan; Seidel, Uwe et al. (2018): Das Technologieprogramm PAiCE des BMWi -Fortschrittsbericht 2018. Hg. v. Institut für Innovation und Technik (iit). Berlin. 
DeVault, David and Artstein, Ron and Benn, Grace and Dey, Teresa and Fast, Ed and Gainer, Alesia and Georgila, Kallirroi and Gratch, Jon and Hartholt, Arno and Lhommet, Margaux and others (2014): SimSensei Kiosk: A virtual human interviewer for healthcare decision support (Proceedings of the 2014 international conference on Autonomous agents and multi-agent systems). Online verfügbar unter http://ict.usc.edu/prototypes/simsensei/. zuletzt geprüft am 22.02.2018.

EFI (2018): Gutachten zu Forschung, Innovation und technologischer Leistungsfähigkeit Deutschlands. Hg. v. Expertenkommission Forschung und Innovation.

El Kaliouby, R.; Robinson, P. (2004): Real-Time Inference of Complex Mental States from Facial Expressions and Head Gestures. In: 2004 Conference on Computer Vision and Pattern Recognition Workshop. 2004 Conference on Computer Vision and Pattern Recognition Workshop. Washington, DC, USA, 27-02 June 2004: IEEE, S. 154.

Feil-Seifer, David; Mataric, Maja (2011): Socially Assistive Robotics. In: IEEE Robot. Automat. Mag. 18 (1), S. 24-31. DOI: 10.1109/MRA.2010.940150.

Graetz, Georg; Michaels, Guy (2015): Robots at work. S.I.: Bureau for Research and Economic Analysis of Development (BREAD working paper, 444). Online verfügbar unter http://ibread.org/bread/working/444, zuletzt geprüft am 22.02.2018.

Greenemeier, Larry (2016): Deadly Tesla Crash Exposes Confusion over Automated Driving. Amid a federal investigation, ignorance of the technology's limitations comes into focus (Scientific American). Online verfügbar unter https://www.scientificamerican.com/article/ deadly-tesla-crash-exposes-confusion-over-automated-driving/, zuletzt aktualisiert am 08.07.2016, zuletzt geprüft am 22.02.2018.

Ikemoto, Shuhei; Amor, Heni; Minato, Takashi; Jung, Bernhard; Ishiguro, Hiroshi (2012): Physical Human-Robot Interaction. Mutual Learning and Adaptation. In: IEEE Robot. Automat. Mag. 19 (4), S. 24-35. DOI: 10.1109/MRA.2011.2181676.

International Federation of Robotics (2017a): World robotics 2017. Industrial robots. [S.I.]: VDMA.

International Federation of Robotics (2017b): World robotics 2017. Service robots. [S.I.]: VDMA.

ISO (2012): ISO 8373:2012: Robots and robotic devices - vocabulary. 2. ed. Geneva: ISO.

Joe Jones (2017): Tertill: A weed whacking robot to patrol your garden. Online verfügbar unter http://robohub.org/tertill-a-weed-whacking-robot-to-patrol-your-garden/, zuletzt aktualisiert am 06.07.2017, zuletzt geprüft am 26.02.2018.

King, Anthony (2017): EU's future cyber-farms to utilise drones, robots and sensors. Online verfügbar unter https://horizon-magazine.eu/article/eu-s-future-cyber-farms-utilise-dronesrobots-and-sensors_en.html, zuletzt geprüft am 26.02.2018.

Lottes, Philipp; Khanna, Raghav; Pfeifer, Johannes; Siegwart, Roland; Stachniss, Cyrill (2017): UAV-based crop and weed classification for smart farming. In: 2017 IEEE International Conference on Robotics and Automation (ICRA). 2017 IEEE International Conference on 
Robotics and Automation (ICRA). Singapore, Singapore, 29.05.2017 - 03.06.2017: IEEE, S. 3024-3031.

Lucas, Gale M.; Rizzo, Albert; Gratch, Jonathan; Scherer, Stefan; Stratou, Giota; Boberg, Jill; Morency, Louis-Philippe (2017): Reporting Mental Health Symptoms. Breaking Down Barriers to Care with Virtual Human Interviewers. In: Front. Robot. Al 4, S. 1017. DOI: 10.3389/frobt.2017.00051.

Mokyr, Joel; Vickers, Chris; Ziebarth, Nicolas (2015): The history of technological anxiety and the future of economic growth. Is this time different? In: The journal of economic perspectives: EP: a journal of the American Economic Association 29 (3), S. 31-50.

Rabe, Benjamin; Kohlbacher, Florian (2015): Pflegerobotik als Innovationstechnik in alternden Gesellschaften - Eine Analyse der Einflussfaktoren auf die Entstehung eines Lead-Markets in Japan. In: Asiatische Studien - Études Asiatiques 69 (2), S. 453. DOI: 10.1515/asia2015-0021.

Seifert, Inessa; Bürger, Matthias; Wangler, Leo; Christmann-Budian, Stephanie; Zinke, Guido (2018): Potenziale der Künstlichen Intelligenz im produzierenden Gewerbe in Deutschland. Eine Studie im Rahmen der Begleitforschung zum Technologieprogramm PAiCE. Hg. v. Institut für Innovation und Technik (iit) in der VDINDE Innovations + Technik GmbH. Berlin.

Sfiligo, Eric (2016): Precision Agriculture: Top 10 technologies. Online verfügbar unter https:// www.therobotreport.com/top-10-technologies-in-precision-agriculture/, zuletzt aktualisiert am 09.09.2016, zuletzt geprüft am 26.02.2018.

Silver, David; Schrittwieser, Julian; Simonyan, Karen; Antonoglou, loannis; Huang, Aja; Guez, Arthur et al. (2017): Mastering the game of Go without human knowledge. In: Nature 550 (7676), S. 354-359. DOI: 10.1038/nature24270.

Sitti, Metin; Ceylan, Hakan; Hu, Wenqi; Giltinan, Joshua; Turan, Mehmet; Yim, Sehyuk; Diller, Eric (2015): Biomedical Applications of Untethered Mobile Milli/Microrobots. In: Proceedings of the IEEE. Institute of Electrical and Electronics Engineers 103 (2), S. 205-224. DOI: 10.1109/JPROC.2014.2385105.

Spitz-Oener, Alexandra (2006): Technical change, job tasks, and rising educational demands. Looking outside the wage structure. In: Journal of labor economics 24 (2), S. 235-270.

Tobe, Frank (2014): Are ag robots ready? 27 companies profiled. Online verfügbar unter https://www.therobotreport.com/are-ag-robots-ready-27-companies-profiled/, zuletzt geprüft am 06.03.2018.

van Henten, E. J.; Hemming, J.; van Tuijl, B.A.J.; Kornet, J. G.; Meuleman, J.; Bontsema, J.; van Os, E. A. (2002): An Autonomous Robot for Harvesting Cucumbers in Greenhouses. In: Autonomous Robots 13 (3), S. 241-258. DOI: 10.1023/A:1020568125418.

Whitney, David; Rosen, Eric; MacGlashan, James; Wong, Lawson L. S.; Tellex, Stefanie (2017): Reducing errors in object-fetching interactions through social feedback. In: 2017 IEEE International Conference on Robotics and Automation (ICRA). 2017 IEEE International 
Conference on Robotics and Automation (ICRA). Singapore, Singapore, 29.05.2017 03.06.2017: IEEE, S. 1006-1013.

Wischmann, Steffen; Hartmann, Ernst Andreas (2018): Zukunft der Arbeit - Eine praxisnahe Betrachtung. Berlin, Heidelberg: Springer Berlin Heidelberg.

Wölbert, Christian (2017): Das sind die Stars auf der Agritechnica. Online verfügbar unter http://www.haz.de/Nachrichten/Wirtschaft/Niedersachsen/Das-sind-die-Stars-auf-derAgritechnica-2017-in-Hannover, zuletzt aktualisiert am 17.11.2017, zuletzt geprüft am 26.02.2018.

\section{(c) (1)}

Dieses Kapitel wird unter der Creative Commons Namensnennung 4.0 International Lizenz http://creativecommons.org/licenses/by/4.0/deed.de) veröffentlicht, welche die Nutzung, Vervielfältigung, Bearbeitung, Verbreitung und Wiedergabe in jeglichem Medium und Format erlaubt, sofern Sie den/die ursprünglichen Autor(en) und die Quelle ordnungsgemäß nennen, einen Link zur Creative Commons Lizenz beifügen und angeben, ob Änderungen vorgenommen wurden.

Die in diesem Kapitel enthaltenen Bilder und sonstiges Drittmaterial unterliegen ebenfalls der genannten Creative Commons Lizenz, sofern sich aus der Abbildungslegende nichts anderes ergibt. Sofern das betreffende Material nicht unter der genannten Creative Commons Lizenz steht und die betreffende Handlung nicht nach gesetzlichen Vorschriften erlaubt ist, ist für die oben aufgeführten Weiterverwendungen des Materials die Einwilligung des jeweiligen Rechteinhabers einzuholen. 